\title{
Descriptive Evaluation of Male Emergency Department Patients in the United States With Gonorrhea and Chlamydia
}

Justin M. Elkins ${ }^{1}$, Santiago Cantillo-Campos ${ }^{1}$, Cheryl Thompson ${ }^{2}$, Michael Mohseni ${ }^{1}$, Johnathan M. Sheele ${ }^{1}$

1. Emergency Medicine, Mayo Clinic, Jacksonville, USA 2. Nutrition, Case Western Reserve University, Cleveland, USA

Corresponding author: Cheryl Thompson, clw8@case.edu

\section{Abstract \\ Introduction}

Sexually transmitted infections are commonly tested for in the emergency department (ED), but diagnostic test results are often unavailable during the clinical encounter.

\section{Methods}

We retrospectively reviewed health records of 3,132 men $\geqslant 18$ years that had an emergency department visit in northeast Ohio between April 18, 2014 and March 7, 2017. All subjects underwent testing for Neisseria gonorrhoeae and Chlamydia trachomatis. Independent $\mathrm{t}$-tests and chi-square analyses were performed as well as multivariable regression analysis.

\section{Results}

On univariable analysis, men with $\mathrm{N}$ gonorrhoeae and/or C trachomatis, compared with uninfected men, were younger (25.9 vs 32.4 years), more likely to be of Black race ( $91.7 \%$ vs $85.6 \%$ ), less likely to be married ( $3.7 \%$ vs $10.2 \%$ ), less likely to arrive to the ED by ambulance or police (1.7\% vs $4.1 \%)$, and more likely to be diagnosed with a urinary tract infection $(8.3 \%$ vs $3.7 \%)$, to be treated for gonorrhea and chlamydia in the ED (84.6\% vs 54.9\%), and to have higher emergency severity index (ESI) scores ( 3.8 vs 3.6$)$ ( $\mathrm{P} \leqslant .03$ for all). On urinalysis, men infected with $\mathrm{N}$ gonorrhoeae and/or $\mathrm{C}$ trachomatis had significantly more white blood cells (55.1 vs 20.9); more mucus (1.3 vs 1.2); higher leukocyte esterase (1.5 vs .4); fewer squamous epithelial cells

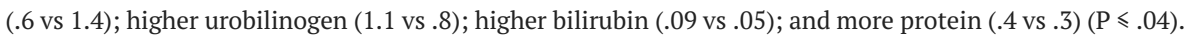

\section{Conclusions}

Demographic and urinalysis findings can be associated with an increased odds of men being infected with $\mathrm{N}$ gonorrhoeae and/or C trachomatis.

Review began 09/16/2020 Review ended 10/21/2020 Published 10/29/2020

(c) Copyright 2020 Elkins et al. This is an open access article distributed under the terms of the Creative Commons Attribution License CC-BY 4.0., which permits unrestricted use, distribution, and reproduction in any medium, provided the original author and source are credited.
Categories: Emergency Medicine, Infectious Disease, Public Health

Keywords: chlamydia trachomatis, emergency department, male sex, neisseria gonorrhoeae, sexually transmitted infection, treatment, antibiotics, emergency medicine, sexually transmitted disease, diagnostic testing

\section{Introduction}

Neisseria gonorrhoeae and Chlamydia trachomatis are among the most common notifiable infectious diseases in the US [1]. In 2018, the prevalence of C trachomatis reached one of the highest levels reported in US history, and the rates of gonorrhea have increased 63\% since 2014 [1-3]. N gonorrhoeae and C trachomatis are increasing primarily among persons 25 years of age or younger and those of Black race [2]. Additional risk factors for $N$ gonorrhoeae and $C$ trachomatis in men include having more sexual partners, having sex with men, and being publicly insured [4,5]. In emergency departments (EDs), the incidence of gonorrhea is more prevalent and increasing among male versus female patients EDs [1].

Nucleic acid amplification tests (NAATs) are the recommended method for diagnosing $N$ gonorrhoeae and $C$ trachomatis [3]. However, most NAAT results are unavailable during the clinical encounter in the ED. Thus, ED clinicians must decide which patients require empiric antibiotic treatment for $\mathrm{N}$ gonorrhoeae and $C$ trachomatis before the testing results are available. Although long-term complications of untreated sexually transmitted infections (STIs) in men are infrequent, they can include penile lymphangitis, penile edema, periurethral abscesses, epididymitis, disseminated gonococcal infection, prostatitis, and post-inflammatory urethral strictures $[3,6]$. Current recommendations are for all patients diagnosed or suspected of having $N$ gonorrhoeae or $C$ trachomatis to be treated if there is a high risk of failure to follow-up as an outpatient $[3,7]$. However, identifying those at risk for loss to follow-up is difficult [8, 9]. Thus, ED patients continue to be overtreated with antibiotics for both $N$ gonorrhoeae and C trachomatis $[7,10]$. 
Men infected with $N$ gonorrhoeae and C trachomatis can have inflammatory changes in the urine including higher levels of urine leukocyte esterase [11-15]. Most research involving $N$ gonorrhoeae and C trachomatis in men come from ambulatory care centers or STI clinics with fewer epidemiologic investigations occurring in the ED patients. The objective of the study was to examine the demographic and clinical variables associated with men infected with $N$ gonorrhoeae and $C$ trachomatis in the ED.

\section{Materials And Methods}

Institutional review board approval was received by University Hospitals, and informed consent was waived for this retrospective study. The dataset included 75,000 ED patient encounters that occurred between April 18, 2014 and March 7, 2017. All patients in the data set were at least 18 years of age and either had a urinalysis and urine culture or were tested for gonorrhea, chlamydia, or trichomonas; however, we only analyzed men who received testing for $N$ gonorrhoeae or $C$ trachomatis. The dataset did not contain patients that were treated but not tested for STIs. Erroneous, incomplete, and missing results were not included in the analysis. An analysis from this dataset has previously been published [16].

Patients were considered infected or uninfected for $N$ gonorrhoeae and $C$ trachomatis on the basis of their Aptima Combo2 NAAT (Hologic, Inc, Marlborough, MA, USA) results. Patients were considered positive for T vaginalis if they had a positive NAAT result or if the parasite was observed on microscopy in the urine or a wet-mount preparation. Patients were considered negative for T vaginalis only if their NAAT results were negative. Patients were considered to have a urinary tract infection (UTI) if their records included an ED International Classification of Diseases (ICD), Ninth Revision or Tenth Revision diagnostic code: N30.90, O23.10, O86.22, N30.00, N30.91, N30, N30.0, N30.01, N30.90, N30.9, N39.0, O23.10, O23.40, O86.20, O08.83, O03.38, O03.88, O04.88, O86.2, O86.29, 595.0, 595.9, 595.89, 599.0, 639.8, 646.60, or 646.64). Triage data was not included in the dataset, but the reason for the ED visit at the initial patient point of contact was recorded. Text searches were performed for the following keywords recorded at the patient ED point of contact using Excel (Microsoft Corp, Redmond, WA, USA). Patients given ceftriaxone or cefixime plus azithromycin or an outpatient prescription for doxycycline were considered treated for $N$ gonorrhoeae and $C$ trachomatis in the ED. Patients given metronidazole either in the ED or as an outpatient prescription were also noted.

Due to laboratory variation in how urine red blood cells (RBCs) and white blood cells (WBCs) were reported, the dataset included either the actual number of cells counted or the mean number of cells if a range was reported. For patients with urine WBC and RBC with $>100$ cells/high-power field (HPF), the value was changed to 101 cells/HPF. The urinalysis included: bacteria (0-4+), bilirubin (0-3+), blood (0-3+), glucose (present or absent), ketones (0-3+ (with “trace” recorded as 0.5$)$ ), leukocyte esterase $(0-3+)$, squamous epithelial cells/HPF, nitrite (positive or negative), protein (0-3+ ("trace" recoded as 0.5 for the analysis)), urobilinogen (<2 (recorded as 0), 2, 4, 8, and $12 \mathrm{mg} / \mathrm{dL}$ ), and urine $\mathrm{pH}(5-9)$.

\section{Data analysis}

Continuous variables were summarized with mean and standard deviation (SD) and analyzed using the independent t-test. Categorical variables were summarized with frequencies and percentages and analyzed using the Fisher's exact test. Stepwise regression analysis was performed using age, race (Black vs nonBlack), type of health insurance, marital status (married/life partner, single, or widowed/separated/divorced), if the patient has a documented primary care physician, emergency severity index score (1-5), and how the patient got to the ED (private vehicle, public transportation, walked, or by police/emergency medical services [EMS]) to identify the variables for a multivariable regression model. The multivariable regression model included age (years), race, and emergency severity index (ESI). Analyses were performed using JMP Pro 14 software (SAS Institute Inc, Cary, NC, USA), and statistical significance was set at an $\alpha$ level of $<.05$.

\section{Results}

Patient characteristics are summarized in Table 1 . There were 3,132 men included in the analysis. A total of $14.6 \%(457 / 3,130)$ of the men tested positive for $C$ trachomatis, $13.3 \%(418 / 3,121)$ tested positive for $N$ gonorrhoeae, and 3.6\% (114/3,132) were coinfected with N gonorrhoeae and C trachomatis. Overall, 24.3\% $(761 / 3,132)$ of men tested in the ED for $N$ gonorrhoeae and/or $C$ trachomatis, had positive results for one or both diseases. The mean age of men undergoing testing for $N$ gonorrhoeae and $C$ trachomatis was 31.0 years (10.9), the majority were of Black race, arrived to the ED by private vehicle, and were single. 


\section{Cureus}

\section{Variable}

Age, mean (SD), y

Race

Black

White

Asian

Other

Method of ED arrival

EMS/police

Public transportation

Car

Walked

Marital status

Married

Life partner

Single

Widowed

Separated

Divorced

Hour of ED visit (0-23)
Mean (SD) or \% (N)

$31.0(10.9)(n=3,132)$

$87.1 \%(2,709 / 3,112)$

$11.2 \%(349 / 3,112)$

$.2 \%(7 / 3,112)$

$1.5 \%(47 / 3,112)$

$3.5 \%(109 / 3,104)$

$2.5 \%(78 / 3,104)$

$91.3 \%(2,834 / 3,104)$

$2.7 \%(83 / 3,104)$

$8.5 \%(267 / 3,131)$

$.03 \%(1 / 3,131)$

$87.7 \%(2,746 / 3,131)$

$.1 \%(4 / 3,131)$

$1.0 \%(30 / 3,131)$

$2.2 \%(68 / 3,131)$

$13.1(6.2)(n=3,132)$

\section{TABLE 1: Patient demographics and triage characteristics}

ED: Emergency department; EMS: Emergency medical services; SD: Standard deviation; y: year.

\section{C trachomatis}

Patients infected with $C$ trachomatis were younger (25.2 vs 31.8 years), of Black (vs not Black) race ( $91.0 \%$ vs $86.4 \%$ ), less likely to be married (2.9\% vs $9.6 \%$ ), had higher emergency severity index (ESI) scores (3.8 vs 3.6), and were more likely to be diagnosed with a UTI (7.9\% vs $4.3 \%)$ than those without $C$ trachomatis on univariable analyses ( $\mathrm{P} \leqslant .006$ for all) (Table 2). Younger age and diagnosed with a UTI were the only significant variables on regression analyses between those with and without $C$ trachomatis $(\mathrm{P}<.001)$.

\begin{tabular}{|c|c|c|c|c|c|}
\hline & $\begin{array}{l}+\mathrm{C} \\
\text { trachomatis }\end{array}$ & $\begin{array}{l}-\mathrm{C} \\
\text { trachomatis }\end{array}$ & $\begin{array}{l}P \\
\text { Value }\end{array}$ & $\begin{array}{l}\text { Adjusted } \\
\text { OR }(95 \% \\
\text { Cl) }\end{array}$ & $\begin{array}{l}\text { Adjusted } \\
\text { P Value }\end{array}$ \\
\hline Age, mean (SD), y & $\begin{array}{l}25.2(6.4)(n \\
=457)\end{array}$ & $\begin{array}{l}31.8(11.2)(n \\
=2,673)\end{array}$ & $\begin{array}{l}< \\
.001\end{array}$ & $\begin{array}{l}.91(.90- \\
.93)\end{array}$ & $<.001$ \\
\hline Black race (vs not Black) & $\begin{array}{l}91.0 \% \\
(414 / 455)\end{array}$ & $\begin{array}{l}86.4 \% \\
(2,293 / 2,655)\end{array}$ & .006 & $\begin{array}{l}1.40(.99- \\
1.99)\end{array}$ & .06 \\
\hline Married/life partner (vs not married) & $\begin{array}{l}2.9 \% \\
(13 / 454)\end{array}$ & $\begin{array}{l}9.6 \% \\
(255 / 2,660)\end{array}$ & $\begin{array}{l}< \\
.001\end{array}$ & $\begin{array}{l}.67(.37- \\
1.22)\end{array}$ & .19 \\
\hline Emergency severity index score, mean (SD), level 1-5 & $\begin{array}{l}3.8(.5)(n= \\
435)\end{array}$ & $\begin{array}{l}3.6(.6)(n= \\
2,524)\end{array}$ & $\begin{array}{l}< \\
.001\end{array}$ & $\begin{array}{l}1.13(.95- \\
1.35)\end{array}$ & .17 \\
\hline Having a (PCP) (vs none) & $\begin{array}{l}16.9 \% \\
(77 / 457)\end{array}$ & $\begin{array}{l}18.8 \% \\
(503 / 2,673)\end{array}$ & .33 & $\begin{array}{l}.84(.63- \\
1.11)\end{array}$ & .21 \\
\hline
\end{tabular}




\section{Cureus}

\begin{tabular}{|c|c|c|c|c|c|}
\hline Arrived to ED by EMS/police (vs other) & $2.0 \%(9 / 455)$ & $\begin{array}{l}3.8 \% \\
(100 / 2,647)\end{array}$ & .05 & $\begin{array}{l}.56(.26- \\
1.19)\end{array}$ & .13 \\
\hline ED triage pain scale, mean $(S D)(0-10)$ & $\begin{array}{l}2.6(3.6)(n= \\
24)\end{array}$ & $\begin{array}{l}3.4(3.8)(n= \\
234)\end{array}$ & .31 & $\begin{array}{l}1.02(.77- \\
1.35)\end{array}$ & .89 \\
\hline $\begin{array}{l}\text { Mentioned "STD," "STI," "sexually transmitted," "penis," or “discharge" at ED point } \\
\text { of contact (vs not) }\end{array}$ & $\begin{array}{l}59.1 \% \\
(270 / 457)\end{array}$ & $\begin{array}{l}45.2 \% \\
(1,208 / 2,673)\end{array}$ & $\begin{array}{l}< \\
.001\end{array}$ & $\begin{array}{l}1.44 \\
(1.15- \\
1.81)\end{array}$ & .002 \\
\hline $\begin{array}{l}\text { Mentioned "UTI," "bladder infection" or "pain," "urinary tract infection," "cystitis," } \\
\text { "dysuria," "frequency" or "frequent urination," or "urgency" at ED point of contact } \\
\text { (vs not) }\end{array}$ & $\begin{array}{l}3.9 \% \\
(18 / 457)\end{array}$ & $\begin{array}{l}4.8 \% \\
(127 / 2,673)\end{array}$ & .55 & $\begin{array}{l}.96(.54- \\
1.70)\end{array}$ & .88 \\
\hline $\begin{array}{l}\text { Mentioned "abdominal pain," "abdominal discomfort," or "abdominal cramping" at } \\
\text { ED point of contact (vs not) }\end{array}$ & $\begin{array}{l}5.0 \% \\
(23 / 457)\end{array}$ & $\begin{array}{l}5.9 \% \\
(158 / 2,673)\end{array}$ & .52 & $\begin{array}{l}1.02(.63- \\
1.65)\end{array}$ & .94 \\
\hline Diagnosed in the ED with a urinary tract infection (vs not) & $\begin{array}{l}7.9 \% \\
(36 / 457)\end{array}$ & $\begin{array}{l}4.3 \% \\
(114 / 2,673)\end{array}$ & .002 & $\begin{array}{l}2.68 \\
(1.75- \\
4.10)\end{array}$ & $<.001$ \\
\hline $\begin{array}{l}\text { Treated with metronidazole in the ED or given it as an outpatient prescription (vs } \\
\text { not) }\end{array}$ & $\begin{array}{l}35.4 \% \\
(162 / 457)\end{array}$ & $\begin{array}{l}30.2 \% \\
(808 / 2,673)\end{array}$ & .03 & $\begin{array}{l}1.12(.90- \\
1.41)\end{array}$ & .31 \\
\hline Treated for gonorrhea and chlamydia (vs not) & $\begin{array}{l}81.2 \% \\
(371 / 457)\end{array}$ & $\begin{array}{l}58.8 \% \\
(1,573 / 2,673)\end{array}$ & $\begin{array}{l}< \\
.001\end{array}$ & $\begin{array}{l}2.67 \\
(2.05- \\
3.47)\end{array}$ & $<.001$ \\
\hline Infected with $\mathrm{N}$ gonorrhoeae (vs not) & $\begin{array}{l}25.1 \% \\
(114 / 455)\end{array}$ & $\begin{array}{l}11.4 \% \\
(304 / 2,664)\end{array}$ & $\begin{array}{l}< \\
.001\end{array}$ & $\begin{array}{l}1.93 \\
(1.49- \\
2.51)\end{array}$ & $<.001$ \\
\hline Infected with T vaginalis (vs not) & $8.0 \%(9 / 112)$ & $\begin{array}{l}8.7 \% \\
(59 / 677)\end{array}$ & $>.99$ & $\begin{array}{l}1.09(.47- \\
2.55)\end{array}$ & .84 \\
\hline \multicolumn{6}{|l|}{ Urinalysis } \\
\hline WBC count (cells/HPF), mean (SD) & $\begin{array}{l}42.1(35.5) \\
(n=198)\end{array}$ & $\begin{array}{l}29.9(37.5)(n \\
=846)\end{array}$ & $\begin{array}{l}< \\
.001\end{array}$ & $\begin{array}{l}1.01(.99- \\
1.05)\end{array}$ & .33 \\
\hline WBC clumps present (vs not) & $\begin{array}{l}6.6 \% \\
(13 / 197)\end{array}$ & $\begin{array}{l}9.3 \% \\
(78 / 837)\end{array}$ & .26 & $\begin{array}{l}.65(.33- \\
1.25)\end{array}$ & .19 \\
\hline Mucus $(0,1+, 2+, 3+$, or $4+)$, mean (SD) & $\begin{array}{l}1.1(1.3)(n= \\
198)\end{array}$ & $\begin{array}{l}1.2(1.1)(n= \\
846)\end{array}$ & .19 & $\begin{array}{l}1.00(.88- \\
1.12)\end{array}$ & .95 \\
\hline Leukocyte esterase $(0,1+, 2+$, or 3+), mean (SD) & $\begin{array}{l}1.1(1.0)(n= \\
246)\end{array}$ & $\begin{array}{l}0.6(1.0)(\mathrm{n}= \\
1,593)\end{array}$ & $\begin{array}{l}< \\
.001\end{array}$ & $\begin{array}{l}1.56 \\
(1.38- \\
1.77)\end{array}$ & $<.001$ \\
\hline Bacteria $(0,1+, 2+, 3+$, or $4+)$, mean (SD) & $\begin{array}{l}0.5(.7)(\mathrm{n}= \\
198)\end{array}$ & $\begin{array}{l}0.6(.9)(n= \\
845)\end{array}$ & .02 & $\begin{array}{l}.91(.74- \\
1.13)\end{array}$ & .41 \\
\hline Nitrite positive (vs negative) & $0.4 \%(1 / 250)$ & $\begin{array}{l}2.1 \% \\
(33 / 1,606)\end{array}$ & .08 & $\begin{array}{l}.43(.06- \\
3.28)\end{array}$ & .41 \\
\hline Blood $(0,1+, 2+$, or 3+), mean (SD) & $\begin{array}{l}0.3(.7)(\mathrm{n}= \\
250)\end{array}$ & $\begin{array}{l}0.3(.7)(n= \\
1,587)\end{array}$ & .92 & $\begin{array}{l}1.17(.96- \\
1.42)\end{array}$ & .11 \\
\hline Red blood cells (cells/HPF), mean (SD) & $\begin{array}{l}9.7(21.8)(\mathrm{n} \\
=198)\end{array}$ & $\begin{array}{l}12.1(24.7)(\mathrm{n} \\
=843)\end{array}$ & .18 & $\begin{array}{l}1.00(.99- \\
1.01)\end{array}$ & .63 \\
\hline Squamous epithelial cells (cells/HPF), mean (SD) & $\begin{array}{l}0.8(3.3)(n= \\
57)\end{array}$ & $\begin{array}{l}1.2(2.5)(n= \\
220)\end{array}$ & .39 & $\begin{array}{l}.98(.87- \\
1.11)\end{array}$ & .74 \\
\hline $\mathrm{pH}(5-9)$, mean (SD) & $\begin{array}{l}6.2(.9)(\mathrm{n}= \\
250)\end{array}$ & $\begin{array}{l}6.0(.9)(\mathrm{n}= \\
1,607)\end{array}$ & .004 & $\begin{array}{l}1.10(.95- \\
1.28)\end{array}$ & .20 \\
\hline Bilirubin $(0,1+, 2+$, or $3+)$, mean (SD) & $\begin{array}{l}0.1(.5)(\mathrm{n}= \\
250)\end{array}$ & $\begin{array}{l}0.04(.3)(n= \\
1,602)\end{array}$ & .06 & $\begin{array}{l}1.51 \\
(1.08- \\
2.11)\end{array}$ & .02 \\
\hline & $1(1.7)(n=$ & $0.8(1.6)(n=$ & & 1.04 (.96- & \\
\hline
\end{tabular}




\section{Cureus}

\begin{tabular}{|c|c|c|c|c|}
\hline Urobilinogen $(0,2,4,8$, or $12 \mathrm{mg} / \mathrm{dL})$, mean (SD) & 250) & $1,606)$ & .01 & 1.13) \\
\hline Protein $(0, .5+, 1+, 2+, 3+)$, mean (SD) & $\begin{array}{l}.4(.6)(n= \\
250)\end{array}$ & $\begin{array}{l}.3(.6)(n= \\
1,606)\end{array}$ & .18 & $\begin{array}{l}1.23(.98- \\
1.54)\end{array}$ \\
\hline Glucose present (vs not) & $.4 \%(1 / 250)$ & $\begin{array}{l}5.2 \% \\
(83 / 1,604)\end{array}$ & $\begin{array}{l}< \\
.001\end{array}$ & $\begin{array}{l}.15(.02- \\
1.12)\end{array}$ \\
\hline Ketones $(0, .5+, 1+, 2+, 3+)$, mean (SD) & $\begin{array}{l}.1(.4)(n= \\
250)\end{array}$ & $\begin{array}{l}.1(.4)(n= \\
1,604)\end{array}$ & .79 & $\begin{array}{l}.97(.66- \\
1.41)\end{array}$ \\
\hline
\end{tabular}

\section{TABLE 2: Demographic and clinical variables and their association with Chlamydia trachomatis in}

men

dL: Deciliter; ED: Emergency department; EMS: Emergency medical services; HPF: High-power field; mg: milligram; NA: Not applicable; OR: Odds ratio; PCP: Primary care physician; SD: Standard deviation; STD: Sexually transmitted disease; STI: Sexually transmitted infection; UTI: Urinary tract infection; vs: versus; WBC: White blood cell; y: year.

Adjusting for age, race, and emergency severity index score. ORs for continuous variables are stated as per unit change in regressor.

On univariable analysis, significant findings between those with and without $C$ trachomatis in the urinalysis were higher WBCs, higher leukocyte esterase, higher urine $\mathrm{pH}$, higher urobilinogen, fewer bacteria, and less likely to have glucose $(\mathrm{P} \leqslant .02)$. On regression analysis only higher leukocyte esterase and urine bilirubin were significant $(\mathrm{P} \leqslant .02)$. There were no significant differences between those with or without $C$ trachomatis and infection with $T$ vaginalis.

\section{N gonorrhoeae}

On univariable analysis, patients infected with $N$ gonorrhoeae were younger (26.4 vs 31.5 years of age), of Black (vs not Black) race (93.0\% vs $86.1 \%$ ), less likely to be married (4.6\% vs 9.3\%), had higher ESI scores (3.8 vs 3.6), were less likely to come to the ED by emergency medical services (EMS) or police (1.5\% vs $3.8 \%$ ), and more likely to be diagnosed with a UTI ( $8.6 \%$ vs $4.2 \%$ ) than those without $N$ gonorrhoeae (P $\leqslant .01$ for all) (Table 3). Younger age, Black race, higher ESI, and diagnosed with a UTI were significant variables on regression analysis ( $\mathrm{P} \leqslant .002$ for all).

Age, mean (SD), y

Black race (vs not Black)

Married/life partner (vs not married)

Emergency severity index score, mean (SD), level 1-5

Having a (PCP) (vs none)

Arrived to ED by EMS/police (vs other)

ED triage pain scale, mean (SD) (0-10)

Mentioned "STD," "STI," "sexually transmitted," "penis," or "discharge" at ED point of contact (vs not)

Mentioned "UTI," "bladder infection" or "pain," "urinary tract infection," "cystitis,"

\begin{tabular}{|c|c|c|c|c|}
\hline $\begin{array}{l}+\mathrm{N} \\
\text { gonorrhoeae }\end{array}$ & $\begin{array}{l}-\mathrm{N} \\
\text { gonorrhoeae }\end{array}$ & $\begin{array}{l}P \\
\text { Value }\end{array}$ & $\begin{array}{l}\text { Adjusted } \\
\text { OR (95\% } \\
\text { Cl) }\end{array}$ & $\begin{array}{l}\text { Adjusted } \\
\text { P Value }\end{array}$ \\
\hline $\begin{array}{l}26.4(7.2)(n= \\
418)\end{array}$ & $\begin{array}{l}31.5(11.2)(\mathrm{n} \\
=2,703)\end{array}$ & $\begin{array}{l}< \\
.001\end{array}$ & $\begin{array}{l}.94(.93- \\
.96)\end{array}$ & $<.001$ \\
\hline $\begin{array}{l}93.0 \% \\
(387 / 416)\end{array}$ & $\begin{array}{l}86.1 \% \\
(2,312 / 2,685)\end{array}$ & $\begin{array}{l}< \\
.001\end{array}$ & $\begin{array}{l}1.90 \\
(1.26- \\
2.85)\end{array}$ & .002 \\
\hline $\begin{array}{l}4.6 \% \\
(19,418)\end{array}$ & $\begin{array}{l}9.3 \% \\
(249 / 2,687)\end{array}$ & .001 & $\begin{array}{l}.96(.58- \\
1.59)\end{array}$ & .86 \\
\hline $\begin{array}{l}3.8(.5)(n= \\
392)\end{array}$ & $\begin{array}{l}3.6(.6)(n= \\
2,558)\end{array}$ & $\begin{array}{l}< \\
.001\end{array}$ & $\begin{array}{l}1.46 \\
(1.21- \\
1.77)\end{array}$ & $<.001$ \\
\hline $\begin{array}{l}17.5 \% \\
(73 / 418)\end{array}$ & $\begin{array}{l}18.7 \% \\
(505 / 2,703)\end{array}$ & .59 & $\begin{array}{l}.91(.69- \\
1.22)\end{array}$ & .54 \\
\hline $1.5 \%(6 / 412)$ & $\begin{array}{l}3.8 \% \\
(103 / 2,681)\end{array}$ & .01 & $\begin{array}{l}.46(.18- \\
1.17)\end{array}$ & .10 \\
\hline $\begin{array}{l}2.4(3.5)(n= \\
27)\end{array}$ & $\begin{array}{l}3.5(3.8)(n= \\
231)\end{array}$ & .17 & $\begin{array}{l}.77(.47- \\
1.26)\end{array}$ & .30 \\
\hline $\begin{array}{l}65.6 \% \\
(274 / 418)\end{array}$ & $\begin{array}{l}44.4 \% \\
(1,201 / 2,703)\end{array}$ & $\begin{array}{l}< \\
.001\end{array}$ & $\begin{array}{l}1.77 \\
(1.39- \\
2.25)\end{array}$ & $<.001$ \\
\hline $4.8 \%$ & $4.6 \%$ & & 1.59 (.95- & \\
\hline
\end{tabular}




\section{Cureus}

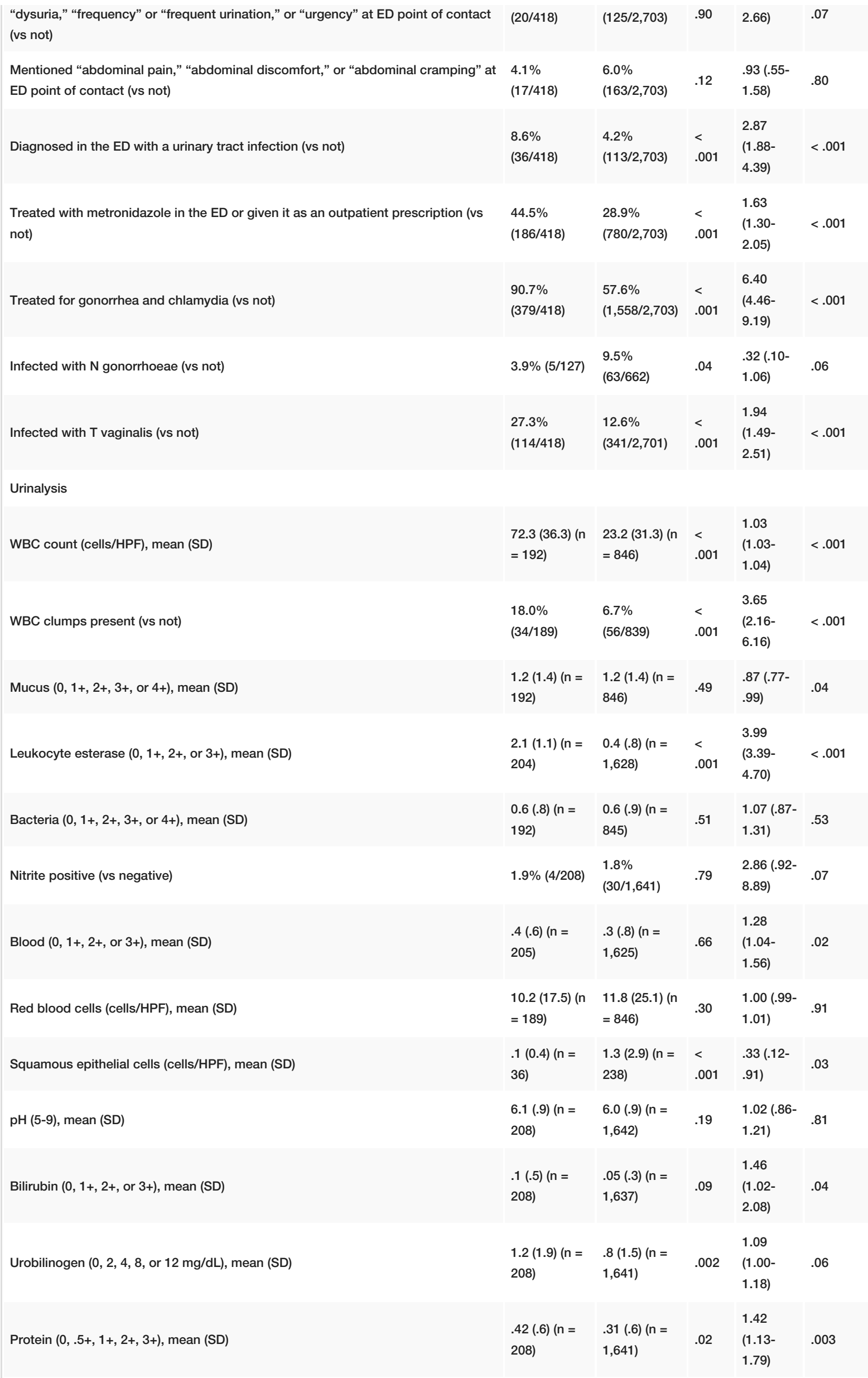




\section{Cureus}

Glucose present (vs not)

Ketones $(0, .5+, 1+, 2+, 3+)$, mean (SD)

\begin{tabular}{|c|c|c|c|}
\hline $2.9 \%(6 / 208)$ & $\begin{array}{l}4.8 \% \\
(78 / 1,639)\end{array}$ & .29 & $\begin{array}{l}.83(.32- \\
2.19)\end{array}$ \\
\hline $\begin{array}{l}.1(.3)(n= \\
208)\end{array}$ & $\begin{array}{l}.1(.4)(n= \\
1,639)\end{array}$ & .16 & $\begin{array}{l}.83(.52- \\
1.32)\end{array}$ \\
\hline
\end{tabular}

\section{TABLE 3: Variables and their association with Neisseria gonorrhoeae in men}

dL: deciliter; ED: Emergency department; EMS: Emergency medical services; HPF: High-power field; mg: milligram; NA: Not applicable; OR: Odds ratio; PCP: Primary care physician; SD: Standard deviation; STD: Sexually transmitted disease; STI: Sexually transmitted infection; UTI: Urinary tract infection; vs: versus; WBC: White blood cell; y: year.

Adjusting for age, race, and emergency severity index score. ORs for continuous variables are stated as per unit change in regressor.

The urinalysis findings that were significantly different between those with and without $\mathrm{N}$ gonorrhoeae on univariable analysis were higher WBCs, more WBC clumps, higher leukocyte esterase, fewer squamous epithelial cells, and higher urobilinogen ( $\mathrm{P} \leqslant .002$ for all). On regression analysis higher WBCs, less mucus, more WBC clumps, higher leukocyte esterase, higher blood, fewer squamous epithelial cells, higher bilirubin, and higher protein were significant $(\mathrm{P} \leqslant .04$ for all).

\section{N gonorrhoeae and/or C trachomatis}

Patients infected with $N$ gonorrhoeae and/or $C$ trachomatis were younger (25.9 vs 32.4 years), of Black race ( $91.7 \%$ vs $85.6 \%$ ), less likely to be married (3.7\% vs $10.2 \%$ ), had higher ESI scores (3.8 vs 3.6), were less likely to come to the ED by EMS or police (1.7\% vs 4.1$)$, and were more likely to be diagnosed with a UTI ( $8.3 \% \mathrm{vs}$ $3.7 \%$ ) on univariable analysis than those uninfected with neither $N$ gonorrhoeae nor $C$ trachomatis ( $\mathrm{P} \leqslant .001$ for all) (Table 4). Younger age, Black race, higher ESI, and diagnosed with a UTI were the significant variables on regression analysis ( $\mathrm{P} \leqslant .001$ for all).

\begin{tabular}{|c|c|c|c|c|c|}
\hline & $\begin{array}{l}+\mathrm{N} \text { gonorrhoeae } \\
\text { and/or C } \\
\text { trachomatis }\end{array}$ & $\begin{array}{l}\text { - N gonorrhoeae } \\
\text { and/or C } \\
\text { trachomatis }\end{array}$ & $\begin{array}{l}\mathbf{P} \\
\text { Value }\end{array}$ & $\begin{array}{l}\text { Adjusted } \\
\text { OR }(95 \% \\
\text { Cl) }\end{array}$ & $\begin{array}{l}\text { Adjusted } \\
\text { P Value }\end{array}$ \\
\hline Age, mean (SD), y & $\begin{array}{l}25.9(6.8)(n= \\
761)\end{array}$ & $\begin{array}{l}32.4(11.4)(n= \\
2,371)\end{array}$ & $\begin{array}{l}< \\
.001\end{array}$ & $\begin{array}{l}.92(.91- \\
.94)\end{array}$ & $<.001$ \\
\hline Black race (vs not Black) & $91.7 \%(695 / 758)$ & $\begin{array}{l}85.6 \% \\
(2,014 / 2,354)\end{array}$ & $\begin{array}{l}< \\
.001\end{array}$ & $\begin{array}{l}1.63 \\
(1.21- \\
2.19)\end{array}$ & .001 \\
\hline Married/life partner (vs not married) & $3.7 \%(28 / 758)$ & $\begin{array}{l}10.2 \% \\
(240 / 2,358)\end{array}$ & $\begin{array}{l}< \\
.001\end{array}$ & $\begin{array}{l}.78(.51- \\
1.20)\end{array}$ & .26 \\
\hline Emergency severity index score, mean (SD), level 1-5 & $3.8(.5)(n=719)$ & $\begin{array}{l}3.6(.7)(n= \\
2,242)\end{array}$ & $\begin{array}{l}< \\
.001\end{array}$ & $\begin{array}{l}1.36 \\
(1.17- \\
1.58)\end{array}$ & $<.001$ \\
\hline Having PCP (vs none) & $17.1 \%(130 / 761)$ & $\begin{array}{l}19.0 \% \\
(451 / 2,371)\end{array}$ & .24 & $\begin{array}{l}.85(.68- \\
1.08)\end{array}$ & .18 \\
\hline Arrived to ED by EMS/police (vs other) & $1.7 \%(13 / 754)$ & $4.1 \%(96 / 2,350)$ & .001 & $\begin{array}{l}.53(.28- \\
1.01)\end{array}$ & .05 \\
\hline ED triage pain scale $(0-10)$, mean (SD) & $2.4(3.5)(n=45)$ & $3.6(3.8)(n=213)$ & .05 & $\begin{array}{l}.92(.73- \\
1.15)\end{array}$ & .45 \\
\hline $\begin{array}{l}\text { Mentioned "STD," "STI," "sexually transmitted," "penis," or "discharge" at } \\
\text { ED point of contact (vs not) }\end{array}$ & $62.4 \%(475 / 761)$ & $\begin{array}{l}42.4 \% \\
(1,005 / 2,371)\end{array}$ & $\begin{array}{l}< \\
.001\end{array}$ & $\begin{array}{l}1.75 \\
(1.45- \\
2.12)\end{array}$ & $<.001$ \\
\hline $\begin{array}{l}\text { Mentioned “UTI," "bladder infection" or "pain," "urinary tract infection," } \\
\text { "cystitis," "dysuria," "frequency" or "frequent urination," or "urgency" at } \\
\text { ED point of contact (vs not) }\end{array}$ & $4.6 \%(35 / 761)$ & $4.6 \%(110 / 2,371)$ & $>.99$ & $\begin{array}{l}1.36(.88- \\
2.09)\end{array}$ & .17 \\
\hline $\begin{array}{l}\text { Mentioned "abdominal pain," "abdominal discomfort," or "abdominal } \\
\text { cramping" at ED point of contact (vs not) }\end{array}$ & $4.1 \%(31 / 761)$ & $6.3 \%(150 / 2,371)$ & .02 & $\begin{array}{l}.82(.54- \\
1.25)\end{array}$ & .36 \\
\hline
\end{tabular}




\section{Cureus}

\begin{tabular}{|c|c|c|c|c|c|}
\hline Diagnosed in the ED with a urinary tract infection (vs not) & $8.3 \%(63 / 761)$ & $3.7 \%(87 / 2,371)$ & $\begin{array}{l}< \\
.001\end{array}$ & $\begin{array}{l}3.67 \\
(2.49- \\
5.41)\end{array}$ & $<.001$ \\
\hline $\begin{array}{l}\text { Treated with metronidazole in the ED or given it as an outpatient } \\
\text { prescription (vs not) }\end{array}$ & $39.9 \%(304 / 761)$ & $\begin{array}{l}28.1 \% \\
(667 / 2,371)\end{array}$ & $\begin{array}{l}< \\
.001\end{array}$ & $\begin{array}{l}1.45 \\
(1.20- \\
1.75)\end{array}$ & $<.001$ \\
\hline Treated for gonorrhea and chlamydia (vs not) & $84.6 \%(644 / 761)$ & $\begin{array}{l}54.9 \% \\
(1,301 / 2,371)\end{array}$ & $\begin{array}{l}< \\
.001\end{array}$ & $\begin{array}{l}4.03 \\
(3.20- \\
5.07)\end{array}$ & $<.001$ \\
\hline Infected with T vaginalis (vs not) & $5.7 \%(12 / 210)$ & $9.7 \%(56 / 580)$ & .09 & $\begin{array}{l}.60(.28- \\
1.29)\end{array}$ & .19 \\
\hline \multicolumn{6}{|l|}{ Urinalysis } \\
\hline WBC count (cells/HPF), mean (SD) & $\begin{array}{l}55.1(38.8)(n= \\
344)\end{array}$ & $\begin{array}{l}20.9(31.1)(n= \\
700)\end{array}$ & $\begin{array}{l}< \\
.001\end{array}$ & $\begin{array}{l}1.03 \\
(1.02- \\
1.03)\end{array}$ & $<.001$ \\
\hline WBC clumps present (vs not) & $11.8 \%(40 / 340)$ & $7.4 \%(51 / 694)$ & .03 & $\begin{array}{l}1.89 \\
(1.13- \\
3.17)\end{array}$ & .02 \\
\hline Mucus $(0,1+, 2+, 3+$, or $4+)$, mean (SD) & $1.3(1.4)(n=344)$ & $1.2(1.3)(n=700)$ & $\begin{array}{l}< \\
.001\end{array}$ & $\begin{array}{l}.94(.85- \\
1.05)\end{array}$ & .28 \\
\hline Leukocyte esterase $(0,1+, 2+$, or $3+)$, mean (SD) & $1.5(1.2)(n=403)$ & $\begin{array}{l}.4(0.8)(n= \\
1,437)\end{array}$ & $\begin{array}{l}< \\
.001\end{array}$ & $\begin{array}{l}2.91 \\
(2.56- \\
3.32)\end{array}$ & $<.001$ \\
\hline Bacteria $(0,1+, 2+, 3+$, or 4+), mean (SD) & $.5(0.8)(\mathrm{n}=344)$ & $.6(0.9)(n=699)$ & .06 & $\begin{array}{l}1.00(.84- \\
1.19)\end{array}$ & .99 \\
\hline Nitrite positive (vs negative) & $1.0 \%(4 / 410)$ & $2.1 \%(30 / 1,447)$ & .21 & $\begin{array}{l}1.25(.40- \\
3.91)\end{array}$ & .70 \\
\hline Blood $(0,1+, 2+$, or $3+)$, mean (SD) & $.3(0.7)(n=407)$ & $\begin{array}{l}.3(0.8)(n= \\
1,431)\end{array}$ & .90 & $\begin{array}{l}1.25 \\
(1.06- \\
1.48)\end{array}$ & .008 \\
\hline Red blood cells (cells/HPF), mean (SD) & $\begin{array}{l}10.0(20.1)(\mathrm{n}= \\
341)\end{array}$ & $\begin{array}{l}12.4(25.9)(n= \\
700)\end{array}$ & .11 & $\begin{array}{l}1.00(.99- \\
1.00)\end{array}$ & .61 \\
\hline Squamous epithelial cells (cells/HPF), mean (SD) & $.6(2.7)(n=85)$ & $1.4(2.6)(n=192)$ & .03 & $\begin{array}{l}.91(.78- \\
1.05)\end{array}$ & .21 \\
\hline pH (5-9), mean (SD) & $6.2(.9)(n=410)$ & $\begin{array}{l}6.0(.9)(n= \\
1,448)\end{array}$ & .007 & $\begin{array}{l}1.07(.94- \\
1.21)\end{array}$ & .33 \\
\hline Bilirubin $(0,1+, 2+$, or $3+)$, mean (SD) & $.09(.4)(n=410)$ & $\begin{array}{l}.05(.3)(n= \\
1,443)\end{array}$ & .04 & $\begin{array}{l}1.42 \\
(1.04- \\
1.94)\end{array}$ & .03 \\
\hline Urobilinogen (0, 2, 4, 8, or $12 \mathrm{mg} / \mathrm{dL})$, mean (SD) & $1.1(1.7)(n=410)$ & $\begin{array}{l}.8(1.5)(n= \\
1,447)\end{array}$ & $\begin{array}{l}< \\
.001\end{array}$ & $\begin{array}{l}1.07(.99- \\
1.14)\end{array}$ & .07 \\
\hline Protein $(0, .5+, 1+, 2+, 3+)$, mean (SD) & $.4(.6)(n=410)$ & $.3(.6)(n=1,447)$ & .02 & $\begin{array}{l}1.34 \\
(1.11- \\
1.62)\end{array}$ & .002 \\
\hline Glucose present (vs not) & $1.5 \%(6 / 410)$ & $5.4 \%(78 / 1,445)$ & $\begin{array}{l}< \\
.001\end{array}$ & $\begin{array}{l}.45(.18- \\
1.17)\end{array}$ & .10 \\
\hline Ketones $(0, .5+, 1+, 2+, 3+)$, mean (SD) & $.1(.3)(n=410)$ & $.1(.4)(n=1,445)$ & .34 & $\begin{array}{l}.91(.66- \\
1.27)\end{array}$ & .59 \\
\hline
\end{tabular}

TABLE 4: Variables and their association with Neisseria gonorrhoeae or Chlamydia trachomatis, or both, in men 


\section{Cureus}

dL: deciliter; ED: Emergency department; EMS: Emergency medical services; HPF: High-power field; mg: milligram; NA: Not applicable; OR: Odds ratio; PCP: Primary care physician; SD: Standard deviation; STD: Sexually transmitted disease; STI: Sexually transmitted infection; UTI: Urinary tract infection; vs: versus; WBC: White blood cell; y: year.

Adjusting for age, race, and emergency severity index score. ORs for continuous variables are stated as per unit change in regressor.

The urinalysis findings that were significantly different between those with $N$ gonorrhoeae and/or $C$ trachomatis, compared with those without $N$ gonorrhoeae and $C$ trachomatis were WBCs, more frequent WBC clumps, more mucus, higher leukocyte esterase level, fewer squamous epithelial cells, higher urine $\mathrm{pH}$, higher bilirubin, higher urobilinogen, higher protein, and less likely to have glucose $(\mathrm{P} \leqslant .04)$. On regression analysis higher WBCs, more WBC clumps, higher leukocyte esterase, higher blood, higher bilirubin, and more protein were significant $(\mathrm{P} \leqslant .03$ for all).

Significantly more patients infected with $N$ gonorrhoeae and/or $C$ trachomatis were treated with antibiotics for the infection in the $\mathrm{ED}(84.6 \%$ vs $54.9 \%)$ than those who had neither infection $(\mathrm{P}<.001)$. Infection with $N$ gonorrhoeae and/or $C$ trachomatis was not significantly associated with infection with $T$ vaginalis.

\section{Discussion}

In this study, $24.3 \%$ of tested men were infected with either $\mathrm{N}$ gonorrhoeae and/or $C$ trachomatis, and $3.6 \%$ were coinfected with $N$ gonorrhoeae and $C$ trachomatis. There were significant differences in the demographics, patient characteristics, and laboratory findings of men with and without $N$ gonorrhoeae and $C$ trachomatis. Younger age, Black race, and unmarried status were all significant risk factors for infection with either $N$ gonorrhoeae and $C$ trachomatis on univariable analysis. Our results are supported by previous research showing that the highest STI infection rates in men were for those aged 20 to 24 years [1]. Being of Black race was a significant risk for $N$ gonorrhoeae and $N$ gonorrhoeae and/or $C$ trachomatis, but not for $C$ trachomatis alone on regression analysis, although race approached significance $(\mathrm{P}=.06)$. Black men were previously found to be 8.5 and 6.8 times more likely to have $N$ gonorrhoeae and $C$ trachomatis than White men [1]. In our study, having a primary care physician (PCP) was not associated with $N$ gonorrhoeae and $C$ trachomatis, which contrasts with other reports showing that women unable to name their PCP were at increased risk for STIs [17].

Patients infected with $N$ gonorrhoeae but not with $C$ trachomatis were associated with $T$ vaginalis coinfection than patients without those infections. Little is known about the epidemiology of $T$ vaginalis in men in the ED $[7,10]$.

On regression analysis, patients with $C$ trachomatis and $N$ gonorrhoeae had a 2.67 and 6.40 odds, respectively for being treated for their respective infections in the ED. This may reflect the higher rates of symptomatic infection in men with $N$ gonorrhoeae than men with C trachomatis [18]. The majority of men (84.6\%) infected with $C$ trachomatis and/or N gonorrhoeae were appropriately treated for the infection in the ED, but $54.9 \%$ were treated and did not have an infection.

There are no externally validated clinical decision rules for men who should undergo testing for $N$ gonorrhoeae and $C$ trachomatis in the ED. Although over a dozen STI prediction models exist, two were developed using ED patients [19,20]. One model used a point system for education, age, marital status, nonantibiotic use in the past month, non-ED as a primary source for health care, new sexual partner within the past two years, dysuria, or discharge in the past three months [21]. The other model was specifically for $N$ gonorrhoeae and $C$ trachomatis in men in the ED and used the following variables: $\leqslant 24$ years of age, penile discharge, lack of health insurance status, and contact with someone infected with $C$ trachomatis or $N$ gonorrhoeae [20].

We identified an association between urine leukocyte esterase and infection with $\mathrm{N}$ gonorrhoeae and $C$ trachomatis on univariable and multivariable regression analyses, and this has previously been suggested to be a screening tool for gonorrhea and chlamydia in asymptomatic men but when used in isolation lacks sufficient sensitivity and specificity [15]. The urine WBC count was associated with $N$ gonorrhoeae and $C$ trachomatis on univariable analysis, but only with $N$ gonorrhoeae on regression analysis. It is possible that these results are related to a stronger inflammatory response caused by $N$ gonorrhoeae compared with $C$ trachomatis in most patients $[13,18,22]$.

\section{Limitations}

All data were retrospective and collected from northeast Ohio. Only data available in the hospital medical record were available for analysis so information on detailed sexual health were unavailable. The dataset also lacked racial diversity. Not all patients were screened for $T$ vaginalis, so the prevalence of coinfection with $T$ vaginalis and $N$ gonorrhoeae and $C$ trachomatis could not be determined. The sensitivity and specificity of the Aptima Combo 2 assay is about $96 \%$ to $99 \%$ sensitive and specific so there are likely some false-positive and negative results in the dataset [13]. Patient history and physical examination 
findings were not included in the dataset, and patients empirically treated for STIs in the ED without testing were not included.

\section{Conclusions}

In the absence of point of care testing clinicians need to decide whether to empirically treat for STIs or wait for the test results. Age, race, being diagnosed with a UTI, and ESI scores plus higher WBCs, higher leukocyte esterase, higher bilirubin, and higher protein on the urinalysis may be helpful when estimating risk for $N$ gonorrhoeae and/or C trachomatis infection in men in the ED. While most men with $N$ gonorrhoeae and $C$ trachomatis were correctly treated with antibiotics in the ED, a majority of patients without $N$ gonorrhoeae and $C$ trachomatis were also inappropriately given antibiotics.

\section{Additional Information \\ Disclosures}

Human subjects: Consent was obtained by all participants in this study. University Hospitals issued approval 18-008945. The study was approved by the University Hospitals institutional review board. Animal subjects: All authors have confirmed that this study did not involve animal subjects or tissue. Conflicts of interest: In compliance with the ICMJE uniform disclosure form, all authors declare the following: Payment/services info: All authors have declared that no financial support was received from any organization for the submitted work. Financial relationships: All authors have declared that they have no financial relationships at present or within the previous three years with any organizations that might have an interest in the submitted work. Other relationships: All authors have declared that there are no other relationships or activities that could appear to have influenced the submitted work.

\section{References}

1. Centers for Disease Control and Prevention. Sexually transmitted disease surveillance 2018 . (2019). Accessed: September 9, 2020: https://www.cdc.gov/std/stats18/default.htm.

2. Torrone E, Papp J, Weinstock H, Centers for Disease Control and Prevention: Prevalence of Chlamydia trachomatis genital infection among persons aged 14-39 years--United States, 2007-2012. MMWR Morb Mortal Wkly Rep. 2014, 63:834-838.

3. Workowski KA, Bolan GA, Centers for Disease Control and Prevention: Sexually transmitted diseases treatment guidelines, 2015. MMWR Recomm Rep. 2015, 64:1-137.

4. Borhart J, Birnbaumer DM: Emergency department management of sexually transmitted infections . Emerg Med Clin North Am. 2011, 29:587-603. 10.1016/j.emc.2011.04.008

5. Pearson WS, Peterman TA, Gift TL: An increase in sexually transmitted infections seen in US emergency departments. Prev Med. 2017, 100:143-144. 10.1016/j.ypmed.2017.04.028

6. Chan PA, Robinette A, Montgomery M, et al.: Extragenital infections caused by Chlamydia trachomatis and Neisseria gonorrhoeae: a review of the literature. Infect Dis Obstet Gynecol. 2016, 2016:5758387. $10.1155 / 2016 / 5758387$

7. Centers for Disease Control and Prevention: Update to CDC's Sexually transmitted diseases treatment guidelines, 2010: oral cephalosporins no longer a recommended treatment for gonococcal infections. MMWR Morb Mortal Wkly Rep. 2012, 61:590-594.

8. Burkins J, DeMott JM, Slocum GW, Gottlieb M, Peksa GD: Factors associated with unsuccessful follow-up in patients undertreated for gonorrhea and chlamydia infections. Am J Emerg Med. 2020, 38:715-719. 10.1016/j.ajem.2019.06.007

9. Schechter-Perkins EM, Jenkins D, White LF, Mitchell PM: Treatment of cases of Neisseria gonorrhoeae and Chlamydia trachomatis in emergency department patients. Sex Transm Dis. 2015, 42:353-357. 10.1097/OLQ.0000000000000299

10. Wegner S, Yen MJ, Witting M: Evidence against the "booty pack": Trichomonas not associated with gonorrhea or chlamydia. J Emerg Med. 2009, 37:124-126. 10.1016/j.jemermed.2007.02.067

11. Bartelsman M, de Vries HJ, Schim van der Loeff MF, Sabajo LO, van der Helm JJ: Leucocyte esterase dip-stick test as a point-of-care diagnostic for urogenital chlamydia in male patients: a multi-center evaluation in two STI outpatient clinics in Paramaribo and Amsterdam. BMC Infect Dis. 2016, 16:625-633. 10.1186/s12879-016-1946-8

12. Ito S, Horie K, Seike K, et al.: Usefulness of quantifying leukocytes in first-voided urine to predict positivity for Chlamydia trachomatis in asymptomatic men at high risk for chlamydial infection. J Infect Chemother. 2014, 20:748-751. 10.1016/j.jiac.2014.08.002

13. McNagny SE, Parker RM, Zenilman JM, Lewis JS: Urinary leukocyte esterase test: a screening method for the detection of asymptomatic chlamydial and gonococcal infections in men. J Infect Dis. 1992, 165:573-576. 10.1093/infdis/165.3.573

14. Rahman MS, Beever W, Skov S, Boffa J: Using urinary leucocyte esterase tests as an indicator of infection with gonorrhoea or chlamydia in asymptomatic males in a primary health care setting. Int J STD AIDS. 2014, 25:138-144. 10.1177/0956462413495670

15. Shafer MA, Schachter J, Moscicki AB, Weiss A, Shalwitz J, Vaughan E, Millstein SG: Urinary leukocyte esterase screening test for asymptomatic chlamydial and gonococcal infections in males. JAMA. 1989, 262:2562-2566.

16. Elkins JM, Hamid OS, Simon LV, Sheele JM: Association of Bartholin cysts and abscesses and sexually transmitted infections (IN PRESS). Am J Emerg Med. 2020, 10.1016/j.ajem.2020.04.027

17. Bachmann LH, Pigott D, Desmond R, et al.: Prevalence and factors associated with gonorrhea and chlamydial infection in at-risk females presenting to an urban emergency department. Sex Transm Dis. 


\section{Cureus}

2003, 30:335-339. 10.1097/00007435-200304000-00012

18. Detels R, Green AM, Klausner JD, et al.: The incidence and correlates of symptomatic and asymptomatic Chlamydia trachomatis and Neisseria gonorrhoeae infections in selected populations in five countries. Sex Transm Dis. 2011, 38:503-509.

19. Falasinnu T, Gustafson P, Hottes TS, Gilbert M, Ogilvie G, Shoveller J: A critical appraisal of risk models for predicting sexually transmitted infections. Sex Transm Dis. 2014, 41:321-330.

10.1097/OLQ.0000000000000120

20. Merchant RC, DePalo DM, Liu T, Rich JD, Stein MD: Developing a system to predict laboratory-confirmed chlamydial and/or gonococcal urethritis in adult male emergency department patients. Postgrad Med. 2010, 122:52-60. 10.3810/pgm.2010.01.2099

21. Al-Tayyib AA, Miller WC, Rogers SM, Leone PA, Gesink Law DC, Ford CA, Rothman RE: Evaluation of risk score algorithms for detection of chlamydial and gonococcal infections in an emergency department setting. Acad Emerg Med. 2008, 15:126-135. 10.1111/j.1553-2712.2008.00027.x

22. Kent CK, Chaw JK, Wong W, Liska S, Gibson S, Hubbard G, Klausner JD: Prevalence of rectal, urethral, and pharyngeal chlamydia and gonorrhea detected in 2 clinical settings among men who have sex with men: San Francisco, California, 2003. Clin Infect Dis. 2005, 41:67-74. 10.1086/430704 